\title{
Structure and dynamics of the ionosphere
}

\section{from A.F. Nagy and L.H. Brace}

A general view of the ionospheric structure of Venus and of the major processes operating there are shown in the figure. Solar extreme ultraviolet (EUV) radiation falling on the thermosphere creates the daytime ionosphere, which is in photochemical equilibrium near its peak at about $142 \mathrm{~km}$, where the major ion is $\mathrm{O}_{2}{ }^{+}$; transport processes become dominant above about $200 \mathrm{~km}$, where the major ion is $\mathrm{O}^{+}$. The upper ionosphere is in near diffusive equilibrium much of the time, although ion transport to the nightside and the loss of plasma through the ionopause frequently cause departures from diffusive equilibrium at high altitudes and in the region near the terminator. A comparison of calculated and measured ion composition indicates that we have achieved a first-order understanding of the very complex photochemical processes involved. Recent suggestions for the inclusion of a large number of specific reactions involving metastable species have even further narrowed the differences between the models and the experimental results. The dayside ionosphere is heated internally by solar EUV-generated photoelectrons and heated from above by the downward conduction of solar wind energy deposited at the ionopause. The ion gas also receives heat from exothermic chemical reactions

A.F. Nagy is Professor of Atmospheric Science at the Space Physics Research Laboratory, University of Michigan, Ann Arbor, Michigan 48109, and L.H. Brace is at the NASA Goddard Space Flight Center, Greenbelt, Maryland 20771. and Joule or frictional heating.

The day-to-night plasma pressure gradient across the terminator drives a nightward flow of ions, usually at supersonic velocities; some of these ions subside to contribute to the formation of the nightside ionosphere. The great variability of the nightside ionosphere is believed to arise from changes in the proportion of the flow which is lost to the wake. Even without the nightward ion flow, a nightside ionosphere would be formed by the precipitation of electrons with energies up to a few hundred electron volts, although the measured electron fluxes do not appear adequate to be the only source of the observed nightside ionization.

Large-scale radial holes or plasma depletions extend downwards to nearly the peak of ionization in the antisolar region. These holes, which occur in north-south pairs, enclose regions of strong radial magnetic fields that are believed to originate in the magnetotail of Venus. The holes or, more precisely, the radial magnetic fields act as a barrier to the flow of ionization into the antisolar region and may be related to the greatly elevated ion temperatures observed there. It has been suggested that the holes are formed by the removal of plasma by outward acceleration caused by magnetotail-generated electric fields.

The ionopause itself is a highly dynamic and complex surface which extends from an average altitude of $290 \mathrm{~km}$ at the subsolar point to about $1,000 \mathrm{~km}$ at the terminator. Its altitude varies from 200 to more than $3,000 \mathrm{~km}$ on the nightside, though its height in the antisolar region will not be measured until further increases occur in the Venus orbiter periapsis altitude during the next few years. The increase in ionopause altitude from the subsolar point towards the terminator reflects the decrease of solar wind dynamic pressure with solar zenith angle. The ionopause altitude is highly variable, with a time constant of probably less than one hour. On the dayside, a near static balance between the solar wind and ionospheric plasma pressures is maintained, which results in an ionopause altitude variation which is inversely proportional to the wind pressure. The solar wind pressure is conveyed to the dayside ionosphere primarily by means of an enhanced magnetic field approximately parallel to the ionopause surface, although some of the solar wind plasma may interact directly with the ionospheric plasma.

Just above the ionopause is the mantle, a transition region between the ionospheric plasma and the flowing shocked solar wind plasma. A variety of solar wind interaction products are observed in the mantle, including suprathermal ions and electrons, ionospheric photoelectrons, enhanced plasma wave activity, and clouds or streamers of ionospheric plasma that appear to be swept downstream by ionosheath flow. These features are probably signatures of the plasma processes that form the ionopause and divert the solar wind about Venus; however the specific processes have not yet been identified.

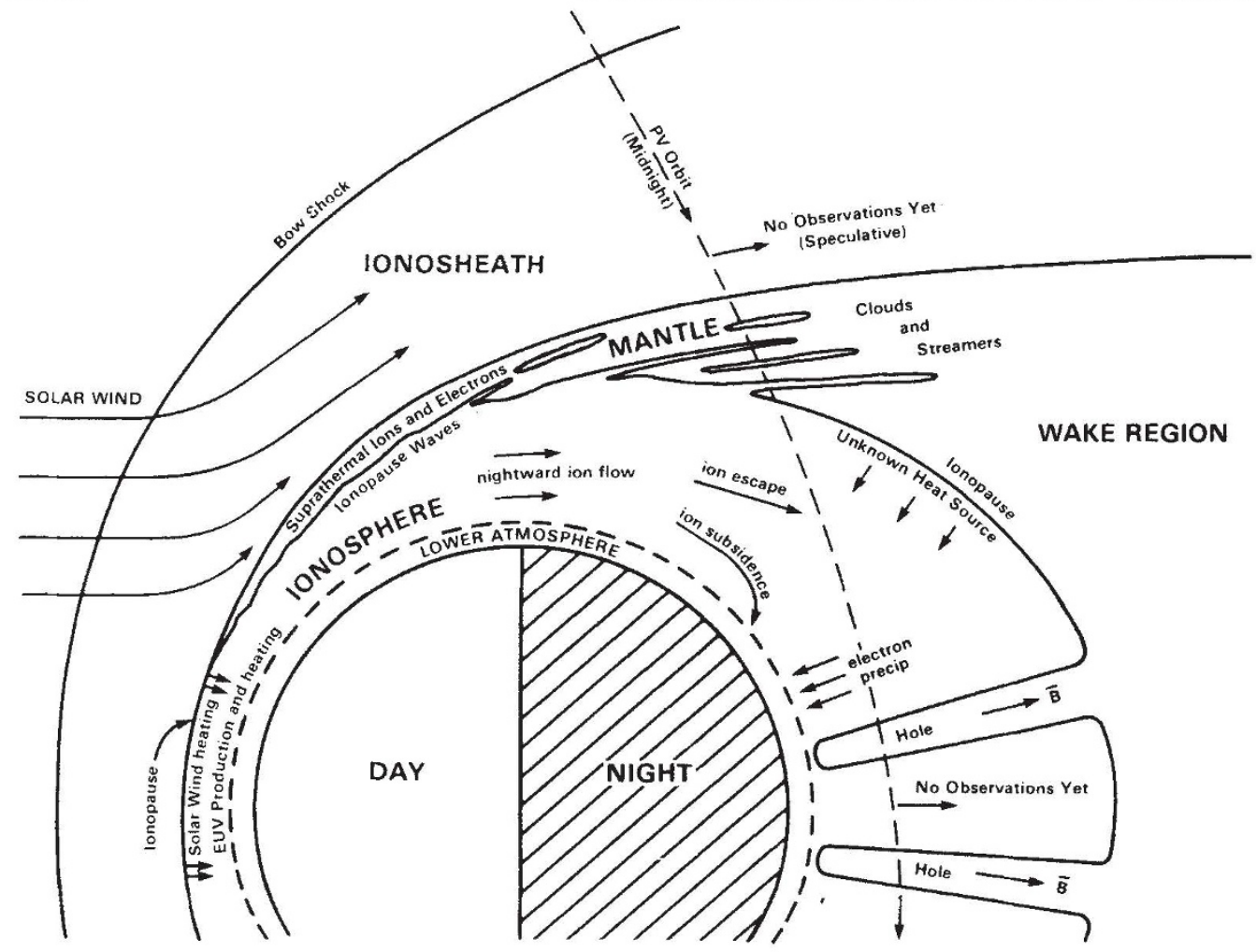

Current views of the ionospheric structure of Venus, showing the major processes that are believed to be operating in the region. The scale of the ionosphere is increased by a factor of three relative to the ionosheath for clarity of presentation (reproduced from Brace, L.H. et al. The Ionosphere of Venus: Observations and their Interpretation, Venus, University of Arizona Press; 1982). 